\title{
RECIPROCAL SERVICE DEPARTMENT COST ALLOCATION AND DECISION MAKING
}

\author{
FRANKLIN LOWENTHAL AND MASSOUD MALEK
}

Received 12 June 2001 and in revised form 10 August 2004

In a manufacturing company, certain departments can be characterized as production departments and others as service departments. Examples of service departments are purchasing, computing services, repair and maintenance, security, food services, and so forth. The costs of such service departments must be allocated to the production departments, which in turn will allocate them to the product. It is known that one can view the cost allocation problem as an absorbing Markov process, with the production departments as the absorbing states and the service departments as the transient states. Using Markov analysis, we will show that this yields additional insight into the underlying concept of reciprocal service department cost allocation by proving that the "full service" department costs can be used to determine the price that should be paid to an external supplier of the same service currently supplied by the service department.

\section{Introduction}

The validity of the linear algebra model to solve the reciprocal service department cost allocation problem has been widely recognized since Kaplan's seminal paper in The Accounting Review in 1973 [1]. Why it is not universally used in the accounting profession and why cost accounting texts as well as the CPA examination persist in still presenting the nonsensical direct and step methods is an embarrassment that one author is addressing in another paper. It is also known that viewing the cost allocation problem as an absorbing Markov process, with the production departments as the absorbing states and the service departments as the transient states, yields additional insight into the underlying concept of reciprocal service department cost allocation. We will show how this approach makes it transparent that the sum of the final costs allocated to all the production departments will always be equal to the sum of all traceable service department costs. In this context, the balancing of the journal entry for the production department cost debits and the service department cost credits will be seen to be a simple consequence of the fact that a Markov process, initially in a transient state, must eventually end in one of the absorbing states. Absorbing Markov chains are also used in accounting to describe an account receivable collection models [2]. Here there are two absorbing states (collected and uncollectible) 
Table 2.1

\begin{tabular}{ll}
\hline Accounts receivable gross & $\$ 1,000,000$ \\
Allowance for uncollectible accounts & $\$ 56,300$ \\
Accounts receivable net & $\$ 943,700$ \\
\hline
\end{tabular}

while the transient states are the various time periods since the account was first recorded such as 30 days, 60 days, 90 days, and so forth. A dollar currently in one of the transient states is absorbed as collected or uncollectible with probabilities that can be determined by an easily understood algorithm. If we denote these probabilities by $p$ and $1-p$, respectively, we can alternatively consider $p$ cents out of each dollar to be collected and $1-p$ cents to be uncollectible. The same methodology can be used to allocate a portion of each service department cost to the respective production departments based on the absorption probabilities [2]. The difference between this model and the accounts receivable model is that there generally are more than two absorbing states as each production department serves as an absorbing state.

\section{Valuation of accounts receivable}

To fairly present the value of the current asset Accounts Receivable on the balance sheet, a business must estimate the portion of the accounts receivable that will never be collected and then deduct this amount from the gross value of accounts receivable. Thus a balance sheet may appear as in Table 2.1.

To determine the estimate for uncollectible accounts, a company will "age" its receivables. Thus it might estimate that $5 \%$ of all 30 -day accounts will not be collected, $10 \%$ of 60 -day accounts and $25 \%$ of 90 -day accounts. By determining the value of the receivables in each category it can then easily estimate the total allowance for uncollectible accounts. How can the company determine the percentages it needs to properly "age" its receivables? Generally, these percentages are based simply on the best guess of the accountant. A more sophisticated model involves viewing this as a Markov process with collection and uncollected as absorbing states and 30 days, 60 days, 90 days, and so forth. as transient states. Historical data is readily available that indicates what portion of receivables in each age category is collected with the next month. Thus each month a receivable in a transient state other than the last is either collected or becomes one month older and thereby moves to the next transient state with known probabilities. From the last transient state a receivable is either collected or written off as uncollectible. For example, if after 150 days a receivable that is not collected is written off, then the last transient state would be 120-day receivables. By the methodology described in Section 4 we can find the probability that a dollar account receivable currently in a particular transient state will never be collected. These probabilities which are found in the matrix $M$ of (4.6) constitute the basis of the percentages used to "age" the accounts receivable.

\section{What is cost allocation?}

In accounting certain costs can be directly traced to a particular product. For example, in a manufacturing firm, direct materials and direct labor refer to material and labor costs 
that can be directly attributed to the product (e.g., wood for a chair). However, nails, sandpaper and plant security personnel all involve costs that cannot be directly traced to a particular product. Nevertheless, it is important in accounting that these costs be allocated to the products that the company manufactures for several reasons. The firm needs to know the "full" cost of the product so that it can determine what an appropriate selling price should be. Further, products that are still in inventory must be properly valued in order to fairly present the balance sheet. Finally, the portion of such costs that are expensed affects net income; costs allocated to a product that remains in inventory are yet to be expensed.

Now accountants universally agree that a cost allocation method is neither right nor wrong but rather reasonable or unreasonable. For example, if all costs of plant security were allocated to one of the three products that a company produced, this would generally be deemed inappropriate and misleading but not wrong. If this product were more valuable than the other two products, then it would be reasonable to allocate more than one third of those costs to the product. However, if the cost accountant nevertheless for simplicity and convenience allocated exactly one third of the cost to the more valuable product, we would say that this was poor choice and might lead to incorrect decision making rather than asserting that it was wrong.

In a manufacturing company, certain departments can be characterized as production departments and others as service departments. Examples of service departments are purchasing, computing services, repair and maintenance, security, food services, and so forth. The costs of such service departments must be allocated to the production departments, which in turn will allocate them to the product. At first sight this appears to permit a straightforward methodology. For example, allocate the costs of food services to the production departments based on the number of personnel employed in the respective production departments. Thus if production department $A$ has $10 \%$ of the production personnel, allocate $10 \%$ of the food services department cost to it. This is reasonable even though it might ignore the fact that the workers in that department eat three meals a day at the cafeteria while those in other departments eat only two. However, this ignores the phenomenon of reciprocal service. The personnel in computing services also eat in the cafeteria; conversely, computing services in all likelihood is responsible for the computing needs of the food services department. If a portion of the cost of the food services department were allocated to computing services, then it would seem appropriate that computing services allocate a portion of its costs right back to food services. Thus we seem to have conjured up the horror of allocating increasing costs back and forth forever among the service departments without ever being able to allocate all the costs to the production departments! Cost accountants refer to this as the reciprocal service cost allocation problem and they have devised several methods for dealing with it. Before describing the one that is mathematically elegant and seemingly most reasonable, we will briefly describe two others that are commonly used. Neither has any basis in logic but they were devised in the dark ages before computers when the solution of a system of linear equations posed a challenge in time to the cost accountant.

The "direct" method ignores the problem created by reciprocal services. If service department $S$ has determined that its costs should be allocated $30 \%$ to production 
department $A, 50 \%$ to production department $B$ and $20 \%$ to service department $T$, simply ignore the $20 \%$ allocation to $T$ and allocate $3 / 8$ of the cost to $A$ and $5 / 8$ of the cost to $B$.

The "step" method is an algorithm that could lead to different results by different users. It would correctly allocate the costs of the above service department $S$ with $20 \%$ of the cost being allocated to $T$. However, no portion of the (increased) cost of $T$ can ever be allocated back to $S$. Thus the cost of $T$ must be allocated by the "direct" method. Clearly, the result depends on the order in which the service department costs are allocated, that is, $S$ followed by $T$ leads to a different bottom line production department allocation then $T$ followed by $S$.

\section{Methodology and notation}

We will assume that there are $n$ service departments and $m$ production departments. Adopting the notation of Minch-Petri [4], we denote by $B=\left(b_{i j}\right)$ the $n \times n$ matrix whose entry $b_{i j}$ in the $i$ th row and $j$ th column is the proportion of the $j$ th service department's output that is provided to the $i$ th service department (we allow the diagonal entries $b_{i i}$ to be strictly positive, i.e., self-service costs). We denote by $C=c_{i j}$ the $m \times n$ matrix whose entry $c_{i j}$ in the $i$ th row and $j$ th column is the proportion of the $j$ th service department's output that is provided to the $i$ th production department. Then for each $j=1,2, \ldots, n$, the sum of the entries in the $j$ th column of $B$ plus the sum of the entries in the $j$ th column of $C$ is equal to one and the sum of the entries in the $j$ th column of $B$ is strictly less than one. Finally, we denote by $b$ the $n \times 1$ column vector whose $i$ th component $b_{i}$ represents the traceable costs of service department $i$. Then the $n \times 1$ column vector $x$ defined by (4.1) below represents the redistributed service department costs after accounting for the interactions among departments (Model 1 in Kaplan's paper):

$$
x=\left(I_{n}-B\right)^{-1} b .
$$

The service department costs allocated to each of the production departments was then given by the $n \times 1$ column vector $v$ determined by (4.2) below where $I_{n}$ represents the $n \times n$ identity matrix:

$$
v=C\left(I_{n}-B\right)^{-1} b .
$$

Kaplan comments that the "full cost" components of the $v$ vector, which clearly must exceed the actual costs of the corresponding component of the $b$ vector have an important economic application: these costs after being divided by the corresponding quantity of output of the respective department, represent the per unit cost that should be paid to an external supplier of the service currently provided by the service department [1]. This assertion is based on two assumptions: (1) All costs are variable; (2) the external supplier will absorb any self-service requirements. We will demonstrate that all this follows easily and elegantly from the theory of absorbing Markov chains. In a Markov process the entries in each row of the transition matrix must sum to one. If a Markov process has $m$ absorbing states and $n$ transient states, then it is well known that by relabeling absorbing 
and transient states, the transition matrix can be expressed in the form below where 0 represents an $m \times n$ matrix of zeros:

$$
S=\left(\begin{array}{cc}
I_{m} & 0 \\
R & Q
\end{array}\right) .
$$

The $n \times m$ matrix $R=\left(r_{i k}\right)$ represents transition probabilities in one period from transient state $i$ to absorbing state $j$ while the square $n \times n$ matrix $Q=\left(q_{i j}\right)$ represents transition probabilities in one period from transient state $i$ to transient state $j$. The fact that the matrix $Q$ is a transition matrix implies that the spectral radius of $Q$ is less than one; this guarantees that the infinite series

$$
I+Q+Q^{2}+Q^{3}+\cdots
$$

converges; its sum, called the fundamental matrix $F$, represents the inverse of the matrix $I_{n}-Q$. Thus we have (4.5) below:

$$
F=\left(f_{i j}\right)=\left(I_{n}-Q\right)^{-1}=I_{n}+Q+Q^{2}+Q^{3}+\cdots
$$

Note that the sum of entries in each row or each column of $F$ is at least one. Finally the product of the $n \times n$ fundamental matrix $F$ and the $n \times m$ matrix $R$, denoted by $M$, is the matrix of absorption probabilities.

$$
M=\left(m_{i j}\right)=F R,
$$

where $M=\left(m_{i j}\right)$ is an $n \times m$ matrix whose $i j$ th entry represents the probability that a Markov process that is initially in transient state $i$ is eventually absorbed in absorbing state $j$. Since eventual absorption is certain, we have that for each $i=1,2, \ldots, m$, the sum of the entries in the $i$ th row of the matrix $M$ is equal to one. In particular, if there is just one absorbing state, then the matrix $M$ is a column vector with all entries equal to one. We will use this last property of the matrix $M$ in our subsequent analysis in Section 5.

\section{Proof of the validity of the linear algebra method}

We consider the production departments to be the absorbing states as once a dollar is allocated to a production department it is never reallocated and the service departments to be the transient states. Using the notation of Section 4 we choose the matrices $Q$ and $R$ as follows:

$$
Q=B^{t}, \quad R=C^{t}
$$

Note that $Q$ and $R$ so defined have the correct Markov properties.

Before considering the main result in this paper, we first briefly repeat the proof of the validity of the linear algebra method based on Markov analysis given in [3]. In this context, validity requires that the total costs allocated to the production departments must equal the total of all traceable costs of the service departments. The rules of matrix 
algebra lead to the following expression for the transpose of the matrix $M$ in (4.6):

$$
\begin{aligned}
& M^{t}=(F R)^{t}=R^{t} F^{t}=C\left[\left(I-B^{t}\right)^{t}\right]^{-1}, \\
& M^{t}=C\left(I_{n}-B\right)_{k}^{-1} .
\end{aligned}
$$

The matrix $M^{t}$ is an $m \times n$ matrix such that for each $j=1,2, \ldots, n$, the sum of the entries in the $j$ th column of $M$ is equal to one.

THEOREM 5.1. The sum of the components of the allocated production department cost vector $v$ given by (4.2) is equal to the sum of the components of the service departments traceable cost vector $b$.

Proof. Combining (4.2) and (5.3) yields

$$
v=M^{t} b .
$$

To sum the $m$ components of the column vector $v$ we can simply multiply the expression of (5.4), $M^{t} b$ on the left by the row vector consisting of $m$ 's, that is, $(1,1,1, \ldots, 1)$. But use the associativity of matrix multiplication to multiply this row matrix by $M^{t}$. This leads to another row matrix but this one has $n$ entries each one of which is just the sum of the entries in the corresponding column of $M^{t}$. But these sum to one. Thus we get another row vector consisting of $n$ ones. Multiplying this matrix by the column vector $b$ gives the sum of the $n$ components of $b$. This proves Theorem 5.1 .

\section{Proof of theorem on "full cost" as cost to pay external supplier}

We assume that all service department costs are strictly variable; thus if the number of units supplied by each service department is represented by the $1 \times n$ row vector $d$, then the traceable cost per unit supplied by the $j$ th service department is just $b_{j} / d_{j}$ while the "full cost" per unit supplied is $x_{j} / d_{j}$. Kaplan's assertion was that were the $j$ th service department eliminated and an external supplier contracted to perform this identical service, then paying this external supplier exactly $x_{j} / d_{j}$ per unit of service will lead to the same total cost to the firm as it presently incurs. Thus this cost per unit represents the indifference point as far as accepting the actual bid of an external supplier. At first this may seem to be too high a price to pay. But further reflection shows that elimination of the service department leads to cost savings in all the other service departments which no longer need supply their services to the department that is eliminated. This leads to even further savings since the reduced level of operations of the other service departments in turn reduces the level of service that they require from the external supplier. Finally, the external supplier is assumed to be responsible for any prior self-service requirements of the service department that is being eliminated.

A major simplification of our analysis is achieved by combining all production departments into one "super" production department. This is a consequence of the fact that there is absolutely no change in any level of service required by a production department as a result of the replacement of the internal service department by an external supplier. Thus $m=1$ and the matrix $C$ is just a $1 \times n$ matrix in our subsequent analysis. 
Without loss of generality we will assume that it is the last service department that is eliminated. The vector $d$ is then replaced by a new unknown vector, denoted by $z$, which represents the reduced level of service that needs to be supplied by each service department and the outside supplier, respectively, $\left(z_{j}\right.$ for $j<n$ and $\left.z_{n}\right)$.

The total cost of contracting the service with an outside supplier with unit price determined by the "full cost vector" $v$ is just:

$$
v=z_{n}\left(\frac{x_{n}}{d_{n}}\right)
$$

while the total savings accruing from the elimination of the service department is just:

$$
b_{n}+\sum_{j=1}^{n-1} b_{j}\left(1-\frac{z_{j}}{d_{j}}\right)
$$

Theorem 6.1. The use of $v_{n}$, the "full cost" of the last service department, will result in the indifference point for the firm, that is, the additional cost of contracting with the outside supplier represented by expression (6.1) is equal to the total cost savings in expression (6.2) that accrues to the firm from eliminating that service department.

Proof. The proof is based on finding an explicit expression for the vector $z$ in terms of the elements of the fundamental matrix.

The original level of service in units provided by the $j$ th service department to the $i$ th service department is just the product $b_{i j} d_{j}=k_{i j} d_{i}$ since the level of service that the $j$ th service department must provide to the $i$ th service department is directly proportional to the size of the $i$ th service department as measured by $d[i]$. The level of service provided by the $j$ th service department to the single production department is just $c_{j} d_{j}$, the elimination of the last service department does not effect the level of service that the $j$ th service department must provide to the production department; it remains $c_{j} d_{j}$. However, the level of service provided by the $j$ th service department to the $i$ th service department for $i<n$ changes to $k_{i j} z_{i}=b_{i j} z_{i} d_{j} / d_{i}$ while the level of service required by the $n$th service department has become zero since it has been eliminated (and its self-service requirements if needed are to be provided by the external supplier). Note that we simply replaced the proportionality constant $k_{i j}$ by its value $b_{i j} z_{i} d_{j} / d_{i}$. If we replace $z_{i} d_{j} / d_{i}$ by $w_{j}$, we are left with the system of $n$ equations for $j=1,2, \ldots, n$ :

$$
\begin{aligned}
& z_{j}=c_{j} d_{j}+\sum_{i=1}^{n-1} b_{i j}\left(\frac{z_{i} d_{j}}{d_{i}}\right)+(0) z_{n}, \\
& w_{j}=c_{j}+\sum_{i=1}^{n-1}\left(b_{i j} w_{i}\right)+(0) w_{n} .
\end{aligned}
$$

If we let $B[n]$ be the matrix $B$ with its last row replaced by zero, the system of $n$ linear equations in $n$ unknowns can be written in matrix form as

$$
\left(I_{n}-B[n]\right) w=C^{t}=R .
$$


144 Service department cost allocation and decision making

If we multiply both sides of (6.4) on the left side by the fundamental matrix $F$, we obtain

$$
F\left(I_{n}-B[n]\right) w=F C^{t}=F R=M
$$

The $n \times 1$ matrix $M$ is just a column vector of ones while the matrix $H=F\left(I_{n}-B[n]\right)$ is an $n \times n$ matrix whose first $n-1$ columns coincide with the identity matrix while its last column is identical to the last column of $F$. This follows directly from the fact that $F$ is the inverse of $I_{n}-B^{t}$ and $(B[n])^{t}$ differs from $B^{t}$ only in that its last column is zero. The system of equations in (6.5) is trivially solvable. We have

$$
w_{n}=\frac{1}{f_{n n}}, \quad \text { and for } j=1,2, \ldots, n-1, w_{j}=1-\frac{f_{j n}}{f_{n n}} .
$$

Since $w_{j}=z_{j} / d_{j}$, the total cost (6.1) of contracting the service from an outside supplier is $x_{n} / f_{n n}$ while the total savings in eliminating the service department (6.2) is

$$
b_{n}+\sum_{j=1}^{n-1} b_{j}\left(\frac{f_{j n}}{f_{n n}}\right)=\sum_{j=1}^{n} b_{j}\left(\frac{f_{j n}}{f_{n n}}\right)
$$

But if we take the transpose of both sides of (4.1) we have

$$
x^{t}=b F
$$

But from (6.8) it is apparent that $x_{n} / f_{n n}$ is precisely the expression in (6.8).

\section{Example}

Algebra Inc. has 4 service departments $S_{1}, S_{2}, S_{3}$, and $S_{4}$ and three production departments $P_{1}, P_{2}$, and $P_{3}$. Direct costs of $\$ 78,000$ for $S_{1}, \$ 200,000$ for $S_{2}, \$ 100,000$ for $S_{3}$ and $\$ 150,000$ for $S_{4}$ are to be allocated to $P_{1}, P_{2}$, and $P_{3}$ by the linear algebra reciprocal service method in accordance with the figures given in Table 7.1.

The above table produces the following matrices:

$$
B=\left(\begin{array}{llll}
0.01 & 0.10 & 0.02 & 0.05 \\
0.04 & 0.05 & 0.02 & 0.02 \\
0.05 & 0.00 & 0.02 & 0.10 \\
0.10 & 0.05 & 0.02 & 0.03
\end{array}\right), \quad C=\left(\begin{array}{llll}
0.45 & 0.20 & 0.32 & 0.20 \\
0.10 & 0.30 & 0.40 & 0.20 \\
0.25 & 0.30 & 0.20 & 0.40
\end{array}\right)
$$

Hence the transpose matrices $Q$ and $R$ are, respectively,

$$
Q=B^{t}=\left(\begin{array}{cccc}
0.01 & 0.04 & 0.05 & 0.10 \\
0.10 & 0.05 & 0.00 & 0.05 \\
0.02 & 0.02 & 0.02 & 0.02 \\
0.05 & 0.02 & 0.10 & 0.03
\end{array}\right), \quad R=C^{t}=\left(\begin{array}{ccc}
0.45 & 0.10 & 0.25 \\
0.20 & 0.30 & 0.30 \\
0.32 & 0.40 & 0.20 \\
0.20 & 0.20 & 0.40
\end{array}\right)
$$


Table 7.1

\begin{tabular}{lcccc}
\hline & $S_{1}$ & $S_{2}$ & $S_{3}$ & $S_{4}$ \\
\hline$S_{1}$ & 40 & 200 & 100 & 500 \\
$S_{2}$ & 160 & 100 & 100 & 200 \\
$S_{3}$ & 0 & 100 & 1000 \\
$S_{4}$ & 200 & 100 & 100 & 300 \\
$P_{1}$ & 400 & 400 & 1600 & 2000 \\
$P_{2}$ & 1800 & 600 & 2000 & 2000 \\
$P_{3}$ & 400 & 600 & 1000 & 4000 \\
\hline Total & 4000 employees & 2000 hours & 5000 calls & 10000 sq. ft. \\
\hline Cost & $\$ 78,000$ & $\$ 200,000$ & $\$ 150,000$ & $\$ 100,000$ \\
\hline Unit cost & $\$ 19.50$ & $\$ 100.00$ & $\$ 30.00$ & $\$ 10.00$ \\
\hline
\end{tabular}

We also have

$$
\begin{gathered}
F=\left(\begin{array}{cccc}
1.02159 & 0.04664 & 0.06324 & 0.10902 \\
0.11056 & 1.05894 & 0.0124 & 0.06623 \\
0.02427 & 0.02310 & 1.02417 & 0.02481 \\
0.05744 & 0.02662 & 0.10910 & 1.04047
\end{array}\right), \\
M=\left(\begin{array}{lll}
0.51109 & 0.16325 & 0.32565 \\
0.27875 & 0.34694 & 0.37429 \\
0.34824 & 0.42399 & 0.22776 \\
0.27418 & 0.26546 & 0.46035
\end{array}\right)
\end{gathered}
$$

To find the cost allocations to the production departments we simply take $M^{t}$ and multiply $4 \times 1$ column vector $b$ of the costs. This results in

$$
v=M^{t} b=\left(\begin{array}{llll}
0.511091 & 0.278756 & 0.348245 & 0.27418 \\
0.163257 & 0.346946 & 0.423993 & 0.265465 \\
0.325652 & 0.374298 & 0.227761 & 0.460355
\end{array}\right)\left(\begin{array}{c}
78000 \\
200000 \\
150000 \\
100000
\end{array}\right)=\left(\begin{array}{c}
175271.09 \\
172268.74 \\
180460.17
\end{array}\right)
$$

Thus the service department costs allocated to each production department:

$$
v=(\$ 175,271.09 \quad \$ 172,268.74 \quad \$ 180,460.17)^{t} .
$$

These allocations add to a total of $\$ 528,000.00$ as advertised in Theorem 5.1.

Next we verify Theorem 6.1 in our example. We assume that $S_{4}$ is replaced by an outside supplier and we replace the three production departments $P_{1}, P_{2}$, and $P_{3}$ by a single super production department whose allocations are the sum of those of $P_{1}, P_{2}$, and $P_{3}$. Hence the matrix $C$ changes into the $1 \times 4$ matrix

$$
C=\left(\begin{array}{llll}
0.80 & 0.80 & 0.92 & 0.80
\end{array}\right)
$$


The 4th component of the original full cost vector

$$
x^{t}=b^{t} F=(\$ 111,182.30 \quad \$ 221,554.43 \quad \$ 171,950.18 \quad \$ 129,520.58)
$$

represents the full cost of service department $S_{4}$. Thus the full cost per square foot of operating service department $S_{4}$ is

$$
\$ 129,520.60 / 10,000=\$ 12.95206 \text { per sq. } \mathrm{ft} .
$$

If $S_{4}$ is replaced by an outside supplier, the reduced service levels required, respectively, by the 3 remaining service departments and the outside supplier are found by first finding the solution of the linear system whose $4 \times 4$ coefficient matrix is the matrix $I_{4}-Q[4]$ where $Q[4]$ is just $Q$ with the last column replaced by zero and the right-hand side is the $4 \times 1$ column vector that is the transpose of $C$. This yields

$$
\left(\begin{array}{llll}
0.895213 & 0.936338 & 0.976154 & 0.961103
\end{array}\right) .
$$

Now multiply the above components by the respective prior service levels or on the left by a $4 \times 4$ diagonal matrix whose diagonal entries are these original service levels.

$$
\left(\begin{array}{l}
3580.853 \\
18726.76 \\
48807.71 \\
9611.028
\end{array}\right)
$$

Thus the outside supplier is paid

$$
[9,611.028 \text { sq. ft. }][\$ 12.95206 \text { per sq. ft. }]=\$ 124,482.60 \text {. }
$$

The savings from the elimination of $S_{4}$ is

$$
\begin{aligned}
{[(4,000} & -3,580.853) \text { employees }][\$ 19.50 \text { per employee }] \\
& +[(20,000-18,726.76) \text { hours }][\$ 10 \text { per hour }] \\
& +[(50,000-48,807.71) \text { calls }][\$ 3 \text { per call }] \\
& +\$ 100,000 \\
= & \$ 124,482.60 .
\end{aligned}
$$

\section{Conclusion}

The entry in the $i$ th row and $j$ th column of the fundamental matrix $F$ represents the mean number of periods that a Markov process initially in the $i$ th transient state spends in the $j$ th transient state before absorption. Thus the diagonal entries of $F$ must be maximum in their respective columns. In other words a system that initially is in transient state $j$ will automatically spend the first period in that state while a system that initially is in a transient state $i \neq j$ must first enter state $j$ before any period can be spent in state $j$. 
Therefore for all $j, f_{j j}>f_{i j}$. In particular, the ratio

$$
\frac{f_{j n}}{f_{n n}}
$$

which is between zero and one, represents the portion of the cost $b_{j}$ of the $j$ th service department that is saved by the firm if the last or $n$th service department is replaced by an outside supplier.

\section{References}

[1] R. S. Kaplan, Variable and self-service costs in reciprocal allocation models, The Accounting Review 48 (1973), no. 4, 738-748.

[2] W. E. Leininger, Quantitative Methods in Accounting, D. Van Nostrand, New York, 1980, 289310.

[3] F. Lowenthal and M. Malek, Cost allocation for outside sources replacing service departments, submitted to Linear Algebra Appl.

[4] R. Minch and E. Petri, Matrix models of reciprocal service cost allocation, The Accounting Review 47 (1972), no. 3, 576-580.

Franklin Lowenthal: California State University, Hayward, CA 94542, USA

E-mail address: flowenth@csuhayward.edu

Massoud Malek: California State University, Hayward, CA 94542, USA

E-mail address: mmalek@csuhayward.edu 


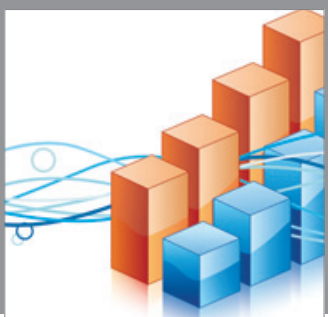

Advances in

Operations Research

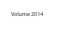

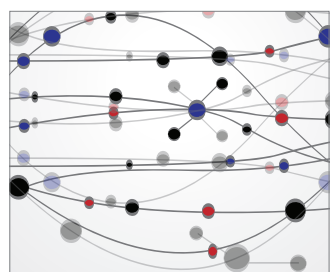

\section{The Scientific} World Journal
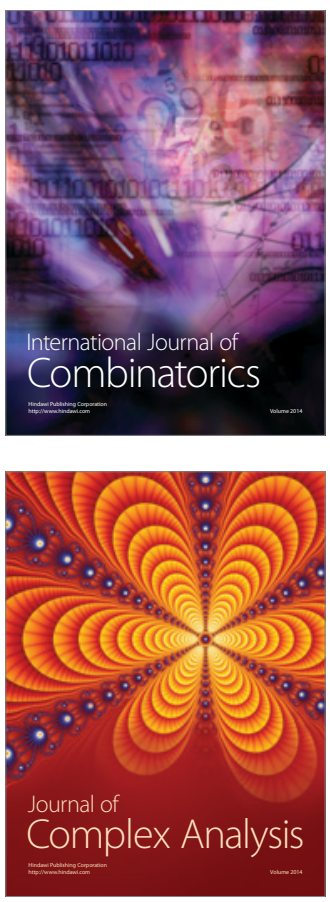

International Journal of

Mathematics and

Mathematical

Sciences
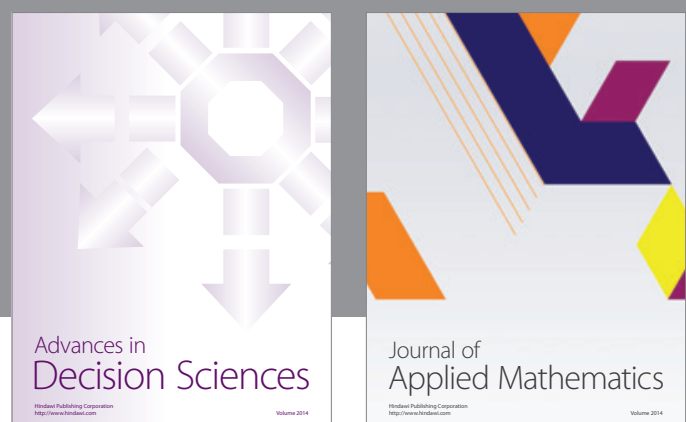

Journal of

Applied Mathematics
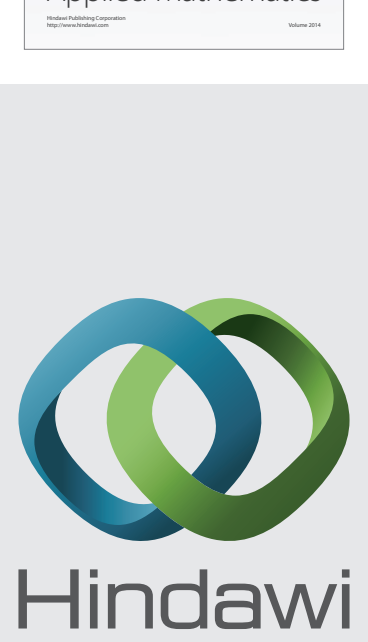

Submit your manuscripts at http://www.hindawi.com
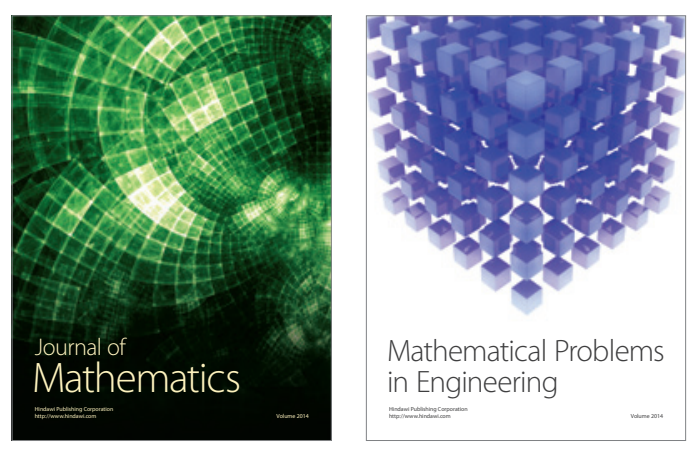

Mathematical Problems in Engineering
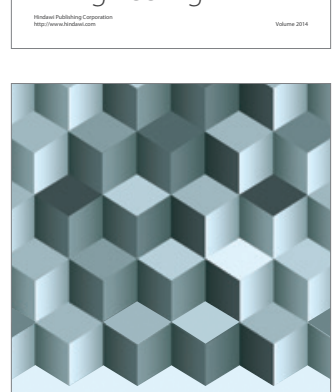

Journal of

Function Spaces
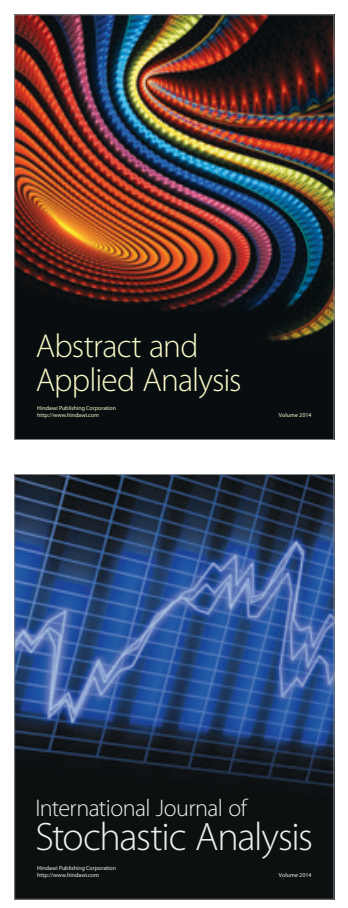

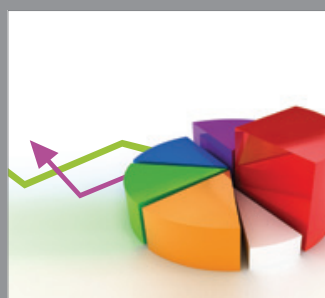

ournal of

Probability and Statistics

Promensencen
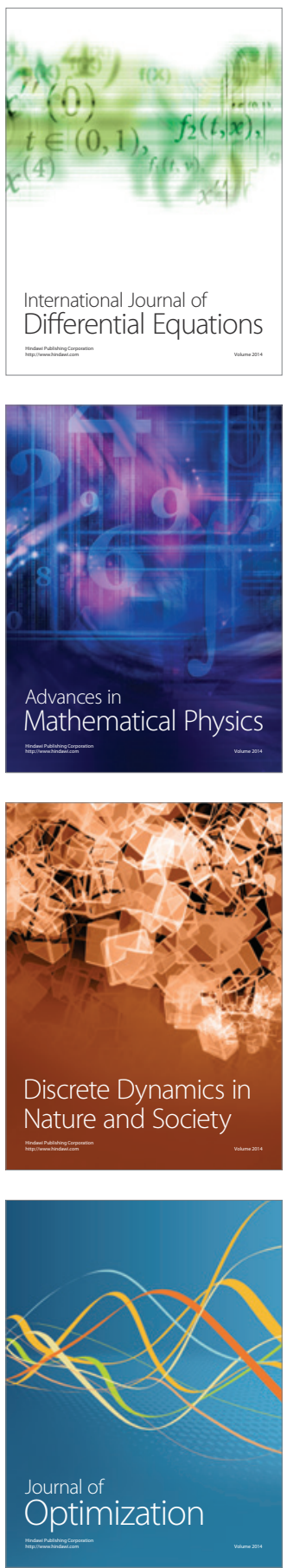